\title{
Novel Applications of Bistable Magnetic Microwires
}

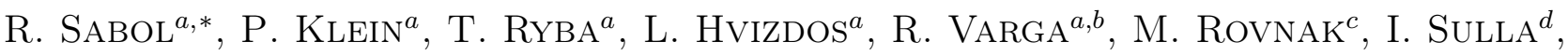

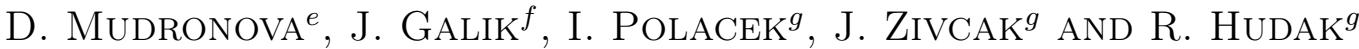 \\ ${ }^{a}$ RVmagnetics, a.s., Hodkovce 21, 04421 Košice, Slovakia \\ ${ }^{b}$ Institute of Physics, Faculty of Sciences, P.J. Safarik University, Park Angelinum 9, 04154 Košice, Slovakia \\ ${ }^{c}$ Faculty of Civil Engineering, TU Košice, Košice 042 00, Slovakia \\ ${ }^{d}$ Dept. Anat. Hist. Phys., UVMP, Komenskeho 74, 04181 Kosice, Slovakia \\ ${ }^{e}$ Dept. Microbiol. Immun., UVMP, Komenskeho 74, 04181 Kosice, Slovakia \\ ${ }^{f}$ Institute of Neurobiology, SAS, Soltesovej 4, 04001 Kosice, Slovakia \\ ${ }^{g}$ Dept. Biomed. Eng. Meas., Fac. Mech. Eng., TU Košice, Košice 042 00, Slovakia
}

\begin{abstract}
Few examples of technical and biomedical applications of bistable magnetic microwires are shown. Particularly, application of microwires in civil engineering are shown for sensing the mechanical stress in concrete, steel structure or steel cable by simple gluing it on the surface of studied structure. On the other hand, biomedical applications for sensing the stress, temperature, and position inside the human or animal body are shown. Experiments with pig intestine cells points to the biocompatibility of glass coated microwires.
\end{abstract}

DOI: 10.12693/APhysPolA.131.1150

PACS/topics: $75.50 . \mathrm{Kj}, 75.60 . \mathrm{Jk}$

\section{Introduction}

Amorphous glass-coated microwires belong to a family of materials with outstanding properties [1]. They are composite materials that consist of metallic core covered by insulating glass cover (Fig. 1). Very cheap preparation technique called the Taylor-Ulitovsky method allows to produce up to few kilometers in a quite short time.
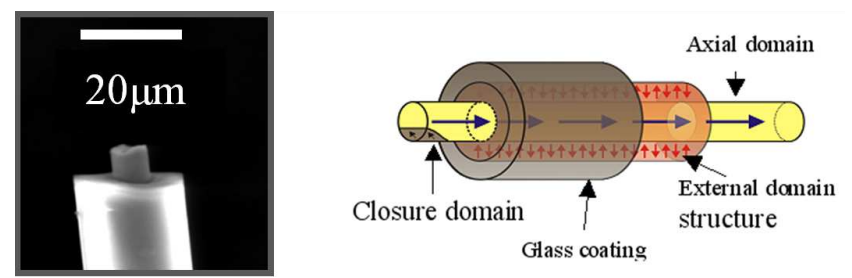

Fig. 1. SEM figure of glass-coated microwire (left). Domain structure of bistable microwire (right).

As a result of magnetoelastic anisotropy, magnetic domain structure consists of large monodomain with axial magnetization in the centre of the wire which is surrounded by radial domain structure (Fig. 1). Magnetization can have only two states + Ms or $-\mathrm{Ms}$ and such property is also called magnetic bistability. The switching between these two states of magnetization runs through domain wall propagation in a single large Barkhausen jump at the field so-called switching field.

The switching field is very sensitive to many external parameters like temperature, magnetic field, frequency or

*corresponding author; e-mail: sabol@rvmagnetics.com mechanical stresses, which can be successfully employed in construction of miniaturized sensors having few advantages in comparison to the currently employed sensors [2].

First of all, the dimension of the wire allows their implementation inside various materials without changing their mechanical properties [3]. Additionally, glasscoating provides insulation from chemically aggressive environment and provides biocompatibility of glasscoated microwires.

In the given contribution, we show few examples of applications of bistable magnetic microwires in civil engineering, electronics and biomedical applications for sensing the stress, temperature and position.

\section{Experimental}

The switching field is usually measured by induction method using the coil system similar to that described in [3]. The system employs two coils: (i) one primary to produce linearly increasing magnetic field, and (ii) one pickup coil to detect the switching field. The advantage of the method is that the switching field is proportional to the time at which the switching is recorded.

Due to the magnetoelastic anisotropy (which is the strongest one in case of positive magnetostriction wire) the switching field is highly sensitive to the stress $\sigma$ applied on metallic nucleus of the wire. The switching field $H_{s w}$ is proportional to the square root of the applied tensile stresses

$$
H_{s w} \sim \sqrt{(A \lambda s \sigma) /\left(\mu_{0} M_{s}\right)},
$$

where $A$ is the constant of exchange length, $\mu_{0}$ is the permeability of vacuum, $M_{s}$ is the saturation magnetization of the metallic nucleus of the wire, $\lambda_{s}$ is saturation magnetostriction and stresses $\sigma$ induced during production process and from different thermal expansion coefficients 
of glass-coating and metallic core $[4,5]$. By this way the switching field $H_{s w}$ varies due to change of applied mechanical load. According to the Hook law, normal stress $\sigma$ is given by equation

$$
\sigma=E \varepsilon,
$$

where $E$ is the Young modulus and $\varepsilon$ is strain of microwires. Stress $\sigma$ depends on strain of microwires because the Young modulus is constant. Applying the triangular shape of excitation magnetic field, the switching field is proportional to the time necessary for switching of domain wall, which is very easy to measure. Finally, the strain dependence of the switching field is obtained showing that the switching time is proportional to the square root of strain

$$
t \approx H_{s w} \approx \varepsilon^{0.5} \text {. }
$$

\section{Results and discussion}

The first application deals with measuring the stress in the concrete structures. First of all, the size of the microwire is smaller than the size of sand grains that form the concrete (inset of Fig. 2). Hence, the microwire does not play the role of the defect in concrete. The compressive stress dependence of the switching field shows the decrease due to the compensation of tensile stress applied on metallic nucleus by glass-coating and follows the square root law given in Eq. (3) (Fig. 2).

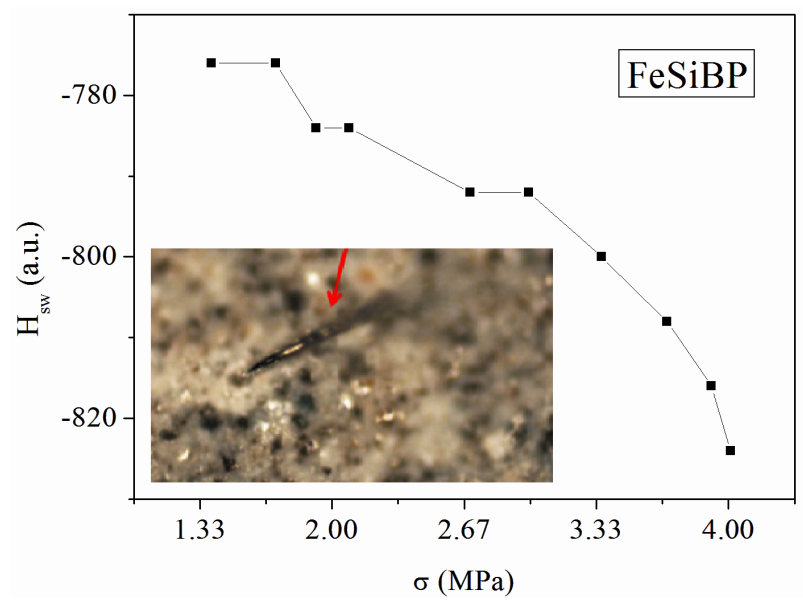

Fig. 2. Compressive stress dependence of the switching field in block of concrete. Inset shows the microwire (emphasized by red arrow) embedded inside the concrete.

Fast magnetization process in bistable microwires (where the domain wall enhances its velocity over $10 \mathrm{~km} / \mathrm{s} \mathrm{[6])} \mathrm{gives} \mathrm{us} \mathrm{advantage} \mathrm{to} \mathrm{sense} \mathrm{the} \mathrm{stress} \mathrm{even} \mathrm{in}$ the case of Fe-based constructions that are magnetic. In the case of magnetic steel, the magnetization processes are spread over wide range of magnetic fields, giving a small induced signal on the pick-up coils. In contrary, fast magnetization process in bistable microwires induces sharp maxima that can be clearly recognized. Figure 3 shows the example of induced maxima on the pick-up coil
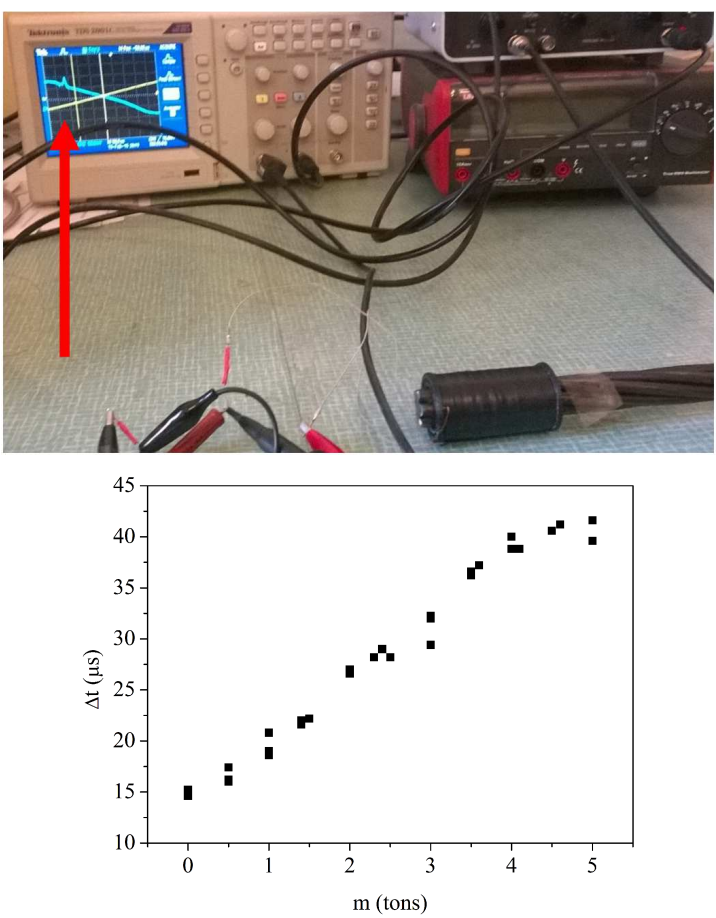

Fig. 3. Example of the sensing microwire glued on the steel cable (up). Red arrow shows the maximum induced on the pick-up coils due to switching in microwire. Dependence of the switching time variation $\Delta t$ on the applied load on steel cable (bottom).

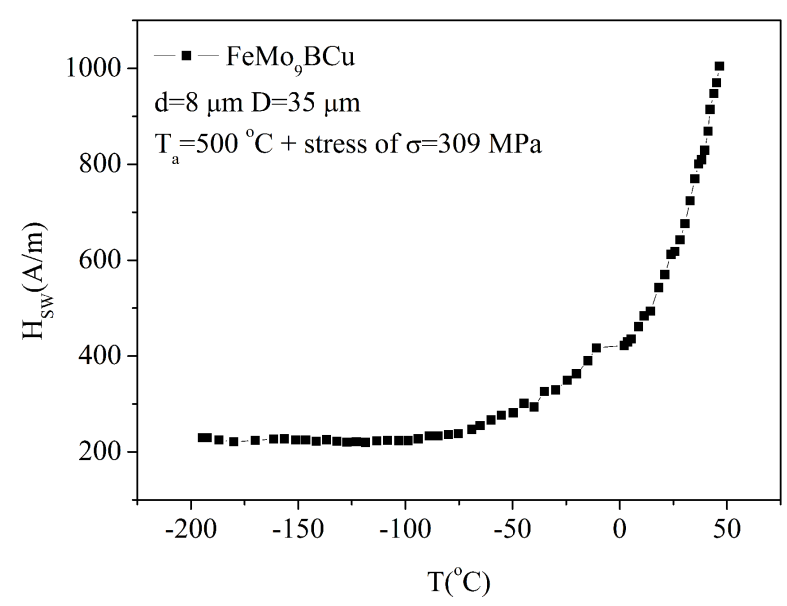

Fig. 4. Temperature dependence of the switching field in nanocrystalline $\mathrm{FeMoBCu}$ microwire.

due to a single domain wall propagation in bistable microwire (shown by red arrows in the upper part of Fig. 3). Lower part of the figure shows the switching time variation dependence on the tensile stress applied on the steel cable (according to Eq. (3)).

Apart from technical applications of bistable microwires, Pyrex glass-coating provides excellent biocompatibility, which gives advantage of use of microwires in bio-applications to sense the stress or temperature in human or animal body. One of the first possible applications is the use of microwire to sense the temperature 
in titanium implants in order to sense the possible inflammation process [7]. In such case, microwires with high sensitivity in a narrow temperature range $\left(35-45^{\circ} \mathrm{C}\right)$ must be employed. Special nanocrystalline microwires with low Curie temperature exhibiting superparamagnetic effect are necessary. Such wires are characterized by sharp increase of coercivity (switching field in case of bistable microwire) just below the Curie temperature, which can reach up to $400 \%$ variation in quite narrow temperature range (Fig. 4). Combining such sensitivity with the high sensitivity of time measurement in oscilloscope, it is possible to reach the sensitivity up to $0.01^{\circ} \mathrm{C}$.

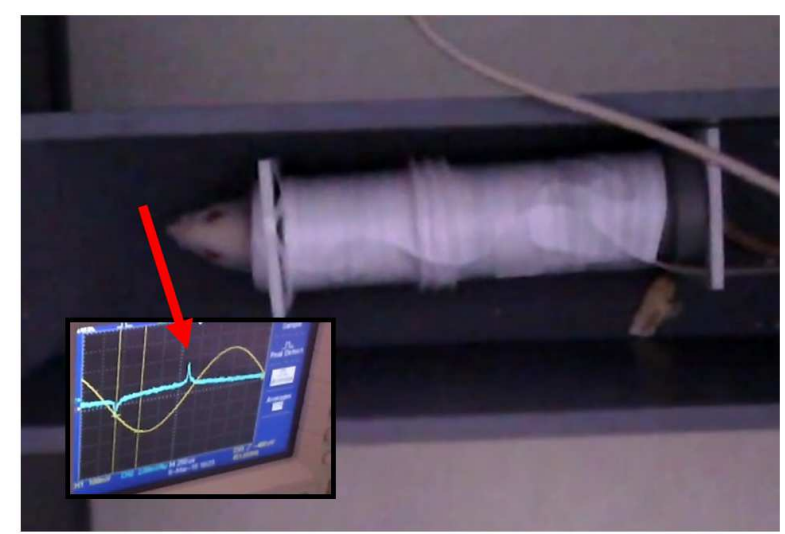

Fig. 5. Example of sensing the microwire inside the rat body. Inset shows the induced signal from the microwire. Arrow points to the maxima due to single domain wall propagation.

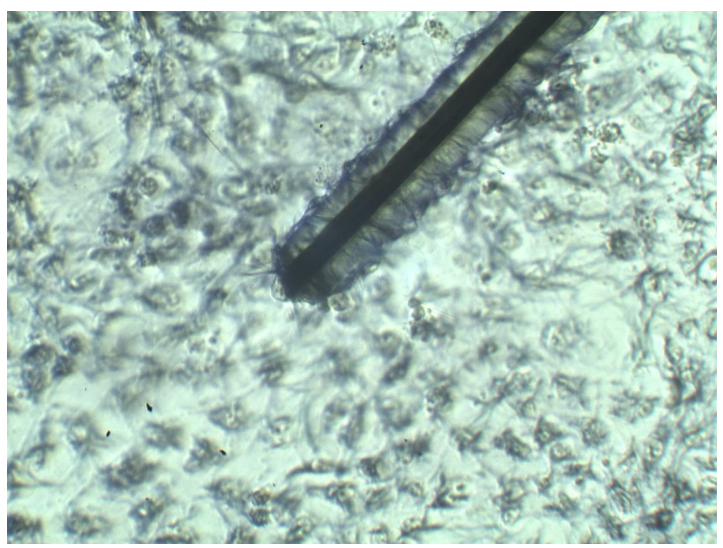

Fig. 6. The glass-coated microwires embedded inside the structure of the pig intestine cells that grew up in the pabulum containing microwire.

Figure 5 shows the example of the microwire application inside the rat body. Clear maxima are induced when the rat crosses the pick-up coils (see inset of Fig. 5). The advantage is that the microwire does not influence the ability to use classical medical treatments like Xray or MRI as it does not (or very weakly) influence their signal [8]. As given above, the glass-coating provides excellent biocompatibility. Figure 6 shows the microwires embedded inside the structure of the pig intestine cells that grew up in the pabulum with the microwire. It is clear that the microwire has diameter comparable to the cell size. Hence, it was incorporated inside the cells structure without producing any defects in their structure. This fact gives us advantage of employing the microwire to sense the stress in muscles, bones and similar body structures.

\section{Conclusions}

Glass-coated microwires are ideal materials for construction of miniaturized sensors (temperature, stress, position etc.) that can be embedded inside of various structures in engineering or biomedical applications. Here, we show few examples of microwires applications in civil engineering for sensing the stress in concrete or in steel cables. On the other hand, microwires can be employed as miniaturized sensors of temperature in biomedicine, where they can be incorporated into the Ti implants or cell structures to provide information on stress or temperature of human or animal body.

\section{Acknowledgments}

This work was partially created with the support of the OP Research and Development for the project: "Completion of the multidisciplinary center for biomedical research - BIOMEDIRES" ITMS 26210120041, cofinanced by the European Regional Development Fund.

\section{References}

[1] M. Vazquez, in: Handbook of Magnetism and Advanced Magnetic Materials, Eds. H. Kronmüller, S. Parkin, Wiley, Chichester, UK 2007, p. 2193.

[2] R. Sabol, M. Rovnak, P. Klein, M. Vazquez, R. Varga, IEEE Trans. Magn. 51, 2000304 (2015).

[3] D. Praslicka, J. Blazek, M. Smelko, J. Hudak, A. Cverha, I. Mikita, R. Varga, A. Zhukov, IEEE Trans. Magn. 49, 128 (2013).

[4] H. Chiriac, T.A. Ovari, Gh. Pop, Phys. Rev. B 52, 10104 (1995).

[5] A.S. Antonov, V.T. Borisov, O.V. Borisov, A.F. Prokoshin, N.A. Usov, J. Phys. D Appl. Phys. 33, 1161 (2000).

[6] P. Klein, R. Varga, M. Vazquez, J. Phys. D Appl. Phys. 47, 255001 (2014).

[7] R. Hudak, R. Varga, I. Polacek, P. Klein, I. Skorvanek, V. Komanicky, R. P. del Real, M. Vazquez, Phys. Status Solidi A 213, 377 (2016).

[8] I. Sulla, R. Sabol, P. Klein, R. Varga, J. Galik, J. Electr. Eng. 66, 161 (2015). 